\begin{tabular}{|c|c|}
\hline Title & $\begin{array}{l}\text { Molecular dynamics simulation of evaporation coefficient of vapor molecules during steady net evaporation in binary } \\
\text { mixture system }\end{array}$ \\
\hline Author(s) & Tabe, Hirofumi; Kobay ashi, Kazumichi; Fujii, Hiroyuki; W atanabe, Masao \\
\hline Citation & $\begin{array}{l}\text { International Journal of Heat and Mass Transfer, } 188,122663 \\
\text { https://doi.org/10.1016/.ijheatmasstransfer.2022.122663 }\end{array}$ \\
\hline Issue Date & 2022-06-01 \\
\hline Doc URL & http:/hdl.handle.net/2115/84072 \\
\hline Rights & $\begin{array}{l}\text { (O) 2022 The A uthors. Published by Elsevier Ltd. This is an open access article under the CC BY-NC-ND license } \\
\text { http://creativecommons.org/icenses/by-nc-nd/4.0/ }\end{array}$ \\
\hline Rights(URL) & http://creativecommons.org/icenses/by-nc-nd/4.0/ \\
\hline Type & article \\
\hline File Information & IJHMT_2022_Tabe.pdf \\
\hline
\end{tabular}

Instructions for use 


\title{
Molecular dynamics simulation of evaporation coefficient of vapor molecules during steady net evaporation in binary mixture system
}

\author{
Hirofumi Tabe*, Kazumichi Kobayashi, Hiroyuki Fujii, Masao Watanabe \\ Division of Mechanical and Space Engineering, Hokkaido University, Kita 13 Nishi 8, Kita-ku, Sapporo, Hokkaido, 080-8628, Japan
}

\section{A R T I C L E I N F O}

\section{Article history:}

Received 23 December 2021

Revised 25 January 2022

Accepted 2 February 2022

\section{Keywords:}

Evaporation coefficient

Non-equilibrium state

Net evaporation

Vapor-gas binary mixture

\begin{abstract}
A B S T R A C T
In this study, an evaporation coefficient representing the evaporation rate of vapor molecules was calculated in a vapor-gas binary mixture and non-equilibrium system using molecular dynamics simulation. The non-equilibrium herein refers to a state in which the net evaporation of vapor molecules occurs, and the calculation system is composed of vapor molecules and non-condensable (NC) gas molecules. The evaporation coefficient in the non-equilibrium state was demonstrated to have almost the same value as that in the equilibrium state. The number density of NC gas molecules around the gas-liquid interface, which is related to the probability that vapor molecules are prevented from evaporating owing to molecular collisions, is shown to be independent of the system state. This makes the evaporation coefficient the same regardless of whether the system is in equilibrium or non-equilibrium. In contrast, the evaporation coefficient decreased as the molar fraction of the NC gas molecules increased. The increase in the molar fraction causes a higher number density of NC gas molecules around the gas-liquid interface, leading to an increase in the number of molecular collisions, which prevents vapor molecules from evaporating. Moreover, it was clarified that the change in the interface structure caused by the presence of NC gas molecules affects the velocity distribution of evaporation vapor molecules.
\end{abstract}

(C) 2022 The Authors. Published by Elsevier Ltd. This is an open access article under the CC BY-NC-ND license (http://creativecommons.org/licenses/by-nc-nd/4.0/)

\section{Introduction}

Molecular gas dynamics (MGD) analyses based on the Boltzmann equation [1,2] enable the investigation of gas flows in the non-equilibrium region in the vicinity of the gas-liquid interface. When conducting MGD analyses of phase-change phenomena, a kinetic boundary condition (KBC) representing the evaporation and condensation of molecules is applied to the interface [3]. In particular, the evaporation coefficient $\alpha_{\mathrm{e}}^{V}$ and the condensation coefficient $\alpha_{c}^{V}$ contained in the KBC for vapor molecules are indispensable parameters for accurately representing the phase change. $\alpha_{\mathrm{e}}^{V}$ and $\alpha_{c}^{V}$ indicate the evaporation and condensation rates, respectively. Although several models have been proposed [3-7], studies on a model defined by the ratio of mass fluxes of molecules moving in the vicinity of the gas-liquid interface have been widely conducted [6,8-14].

\footnotetext{
* Corresponding author.

E-mail address: azx@eis.hokudai.ac.jp (H. Tabe).
}

The definitions of $\alpha_{\mathrm{e}}^{V}$ and $\alpha_{\mathrm{c}}^{V}$, given by the ratio of the molecular mass fluxes, are as follows:

$\alpha_{\mathrm{e}}^{V}=\frac{J_{\text {evap }}^{V}}{J_{\text {out }}^{V *}}, \quad \alpha_{\mathrm{c}}^{V}=\frac{J_{\text {cond }}^{V}}{J_{\text {coll }}^{V}}$,

where $J_{\text {out }}^{V *}$ denotes the mass flux of vapor molecules outgoing from the gas-liquid interface to the gas phase in the equilibrium state, $J_{\text {evap }}^{V}$ is the mass flux of evaporation vapor molecules, $J_{\text {coll }}^{V}$ is the mass flux of the vapor molecules colliding with the interface, and $J_{\text {cond }}^{V}$ is the mass flux of the condensation vapor molecules. Fig. 1 shows the relations between molecular mass fluxes given by the following equations:

$J_{\text {out }}^{V}=J_{\text {evap }}^{V}+J_{\text {ref }}^{V}, J_{\text {coll }}^{V}=J_{\text {ref }}^{V}+J_{\text {cond }}^{V}$,

where $J_{\text {out }}^{V}$ is the mass flux of vapor molecules outgoing from the interface to the gas phase, and $J_{\text {ref }}^{V}$ is the mass flux of the vapor molecules reflected in the vicinity of the interface to return to the gas phase. To calculate $\alpha_{\mathrm{e}}^{V}$ and $\alpha_{\mathrm{c}}^{V}$, molecular-scale simulation methods have been used because obtaining the molecular mass fluxes from molecules irregularly moving around the interface is necessary. 


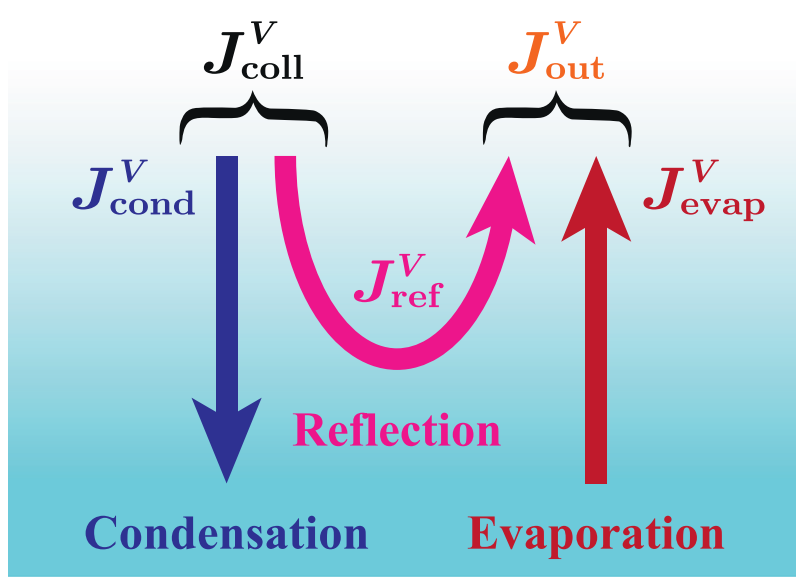

Fig. 1. Relation between molecular mass fluxes in the vicinity of the gas-liquid interface.

Ishiyama et al. have conducted a single-component molecular dynamics (MD) simulation [6] to calculate the evaporation coefficient $\alpha_{\mathrm{e}}^{V}$ in a virtual vacuum condition where the condensation of vapor molecules does not occur and only evaporation into vacuum occurs. In such a condition, net evaporation occurs when the evaporation amount is larger than the condensation amount. They demonstrated that the evaporation mass flux during the net evaporation hardly change from that in the equilibrium state where the evaporation amount was equal to the condensation amount. From this result, they proposed spontaneous evaporation, where the evaporation mass flux is a function of only the liquid temperature [15]. This indicates that $\alpha_{\mathrm{e}}^{V}$, which can be calculated from the evaporation mass flux, is also a function of the liquid temperature, and $\alpha_{\mathrm{e}}^{V}$ in the single-component system takes the same value regardless of whether the system is in the equilibrium state or the non-equilibrium state where the net evaporation occurs. Based on the concept of spontaneous evaporation, some studies have been conducted to calculate $\alpha_{\mathrm{e}}^{V}$ and $\alpha_{\mathrm{c}}^{V}$ under various system conditions [8,9,12-14,16-21].

Recently, $\alpha_{\mathrm{e}}^{V}$ and $\alpha_{\mathrm{c}}^{V}$ were calculated in a vapor-gas binary mixture system composed of vapor molecules and noncondensable (NC) gas molecules [14,21]. In addition, the effect of non-condensed molecules on the evaporation of vapor molecules has been investigated in the form of a mass accommodation coefficient (MAC), where $\alpha_{\mathrm{e}}^{V}=\alpha_{\mathrm{c}}^{V}$ holds [22-24]. In a binary mixture system, $\alpha_{\mathrm{e}}^{V}$ and $\alpha_{\mathrm{c}}^{V}$ of vapor molecules are defined as functions of the molar fraction of $\mathrm{NC}$ gas molecules in the liquid phase as well as the liquid temperature [25]. Although $\alpha_{\mathrm{e}}^{V}$ and $\alpha_{\mathrm{c}}^{V}$ in the binary mixture system have been calculated under certain conditions of liquid temperature [14] or partial pressure of NC gas molecules $[14,21]$, they have not been calculated in the non-equilibrium state. As some multi-component MGD analyses have been performed for non-equilibrium and phase-change phenomena [26-28], calculating $\alpha_{\mathrm{e}}^{V}$ and $\alpha_{\mathrm{c}}^{V}$ in the binary and non-equilibrium systems is desirable.

The non-equilibrium indicates the state in which the evaporation amount and condensation amount do not match. Hence, the non-equilibrium state is classified into two types: the state in which the evaporation amount is larger and the state in which the condensation amount is larger. Net evaporation occurs under the former condition, as mentioned earlier, whereas net condensation occurs under the latter condition.
In this study, we conducted MD simulations to calculate the $\alpha_{\mathrm{e}}^{V}$ of vapor molecules during steady net evaporation in a binary mixture system. In particular, we proposed a computational system that can perform net evaporation calculations up to the extreme case of non-equilibrium, and investigated in detail the values of $\alpha_{\mathrm{e}}^{V}$ and the evaporation mechanisms in several system states. Based on spontaneous evaporation under the virtual vacuum condition in a single-component system [6], we focused on the nonequilibrium state wherein condensation of vapor molecules does not occur and only evaporation occurs. By calculating $\alpha_{\mathrm{e}}^{V}$ in such a non-equilibrium state, we can clarify whether $\alpha_{\mathrm{e}}^{V}$ in the nonequilibrium state where the net evaporation occurs takes the same value as in the equilibrium state even in the binary mixture system. We also investigated the influence of the molar fraction of the NC gas molecules in the liquid phase on $\alpha_{\mathrm{e}}^{V}$. From the above, the objectives of this study are summarized as follows: to calculate $\alpha_{\mathrm{e}}^{V}$ in the non-equilibrium and binary mixture system to elucidate the influences of the system states and the presence of NC gas molecules on $\alpha_{\mathrm{e}}^{V}$ and to clarify the mechanisms by which these factors affect $\alpha_{\mathrm{e}}^{V}$.

In the binary mixture system, the non-equilibrium state without condensation indicates a state wherein evaporation into the gas phase composed only of NC gas molecules occurs instead of evaporation into vacuum. As the objective of this study is to calculate $\alpha_{\mathrm{e}}^{V}$ in the non-equilibrium state caused by the mismatch between the evaporation and condensation amounts, other conditions such as the system temperature and states of NC gas molecules were controlled to remain constant as those in the equilibrium state. Thus, the steady and net evaporation of vapor molecules under constant conditions was reproduced in the current MD simulations.

\section{Method}

\subsection{Calculation system and potential functions}

The calculation system was composed of Ar molecules as vapor molecules and Ne molecules as NC gas molecules. To investigate the influence of $\mathrm{NC}$ gas molecules on the evaporation of vapor molecules, simulations were performed in four cases with different numbers of Ne molecules. The number of Ne molecules, $N_{\mathrm{Ne}}$, was set to $N_{\mathrm{Ne}}=0,4,000,8,000$, and 12,000, while the number of $\mathrm{Ar}$ molecules was fixed at 12,000.

Figs. 2(a) and 3(a) show the calculation systems in the cases of $N_{\mathrm{Ne}}=4,000$ and 12,000, respectively. Blue spheres represent $\mathrm{Ar}$ molecules and yellow spheres represent Ne molecules. The liquid film was formed at the center of the calculation system, and gas phases were formed on both sides of the film. The lengths of the system $L_{x}, L_{y}$, and $L_{z}$ were $8.0,8.0$, and $175.0 \mathrm{~nm}$, respectively. In this study, we set $L_{z}$, which is considerably longer than $L_{x}$ and $L_{y}$, the aim of which is explained in Section 2.4. The periodic boundary condition was imposed in all directions of the system.

For the intermolecular potential, the following 12-6 LennardJones potential function was utilized:

$\varphi(r)=4 \varepsilon\left[\left(\frac{\sigma}{r}\right)^{12}-\left(\frac{\sigma}{r}\right)^{6}\right]$,

where $\sigma$ denotes the molecular diameter, $\varepsilon$ denotes the potential depth, and $r$ denotes the distance between two molecules. $\sigma_{\mathrm{Ar}}=0.3405 \mathrm{~nm}$ and $\varepsilon_{\mathrm{Ar}} / k_{\mathrm{B}}=119.8 \mathrm{~K}$ were applied to the $\mathrm{Ar}$ $\mathrm{Ar}$ intermolecular potential, and $\sigma_{\mathrm{Ne}}=0.2750 \mathrm{~nm}$ and $\varepsilon_{\mathrm{Ne}} / k_{\mathrm{B}}=$ $35.05 \mathrm{~K}$ were applied to $\mathrm{Ne}-\mathrm{Ne}\left(k_{\mathrm{B}}\right.$ denotes the Boltzmann constant). In addition, the following parameters derived from the Lorentz-Berthelot mixing rules were applied to the Ar-Ne inter- 


$$
N_{\mathrm{Ne}}=4,000
$$

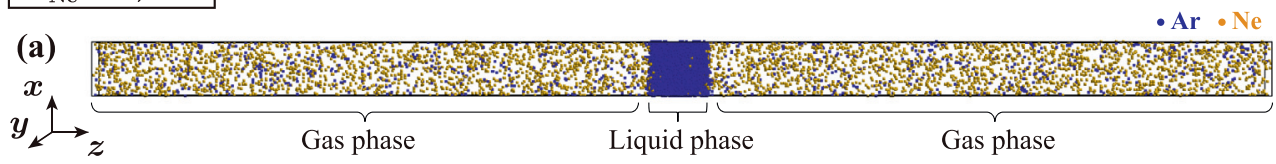

(b)

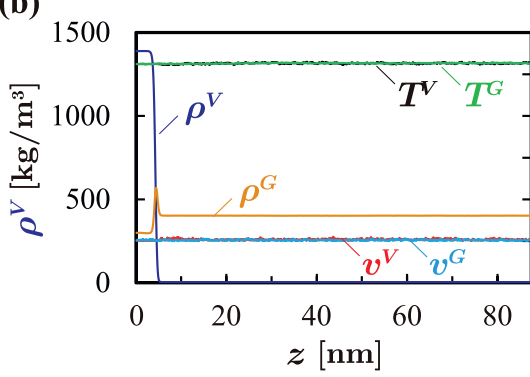

(c)

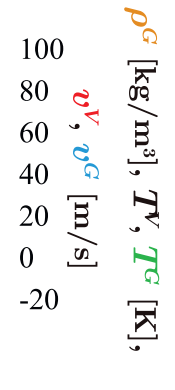

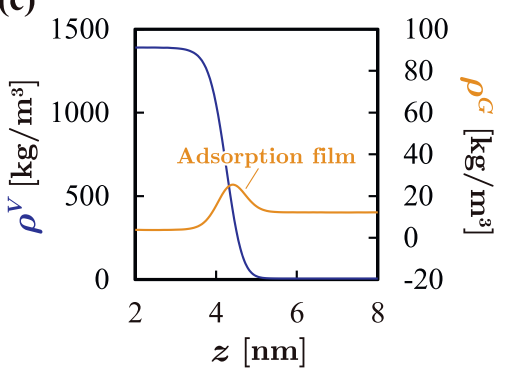

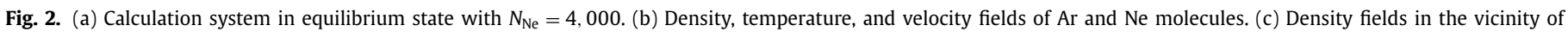
the gas-liquid interface $(2 \leq z \leq 8)$.

$$
N_{\mathrm{Ne}}=12,000
$$

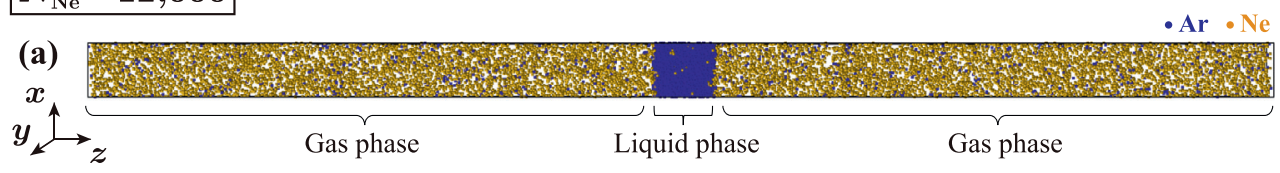

(b)

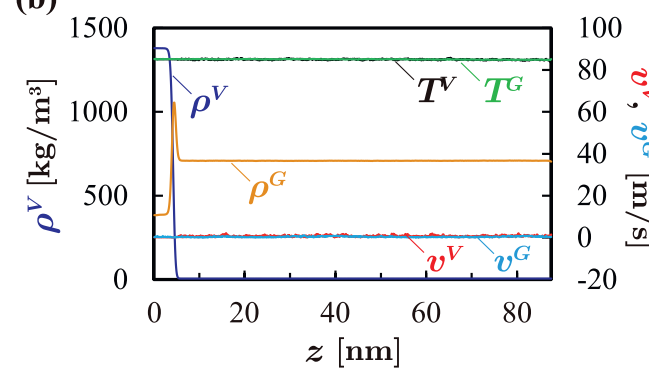

(c)

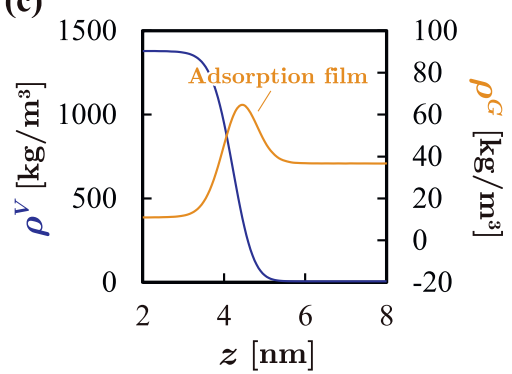

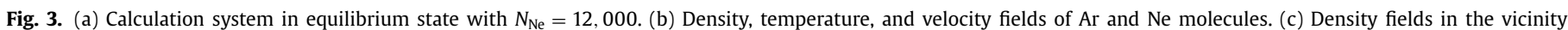
of the gas-liquid interface $(2 \leq z \leq 8)$.

molecular potential:

$\sigma_{\mathrm{Ar}-\mathrm{Ne}}=A \frac{\sigma_{\mathrm{Ar}}+\sigma_{\mathrm{Ne}}}{2}, \varepsilon_{\mathrm{Ar}-\mathrm{Ne}}=B \sqrt{\varepsilon_{\mathrm{Ar}} \cdot \varepsilon_{\mathrm{Ne}}}$.

To satisfy Henry's law, which describes the relation between the dissolved amount and partial pressure of gas molecules, we set $A=1.01903$ and $B=0.89554$ [29]. The same setting of the potential functions was applied in a previous study [14]. The cutoff radius was set as $1.5 \mathrm{~nm}$, and Newton's law of motion was solved by the leapfrog method with the time step $\Delta t=5 \mathrm{fs}$. The system temperature was set to $T=85 \mathrm{~K}$.

When establishing the equilibrium system for the initial state of the present MD simulations, the velocity scaling method [30] was applied to all molecules to maintain the system temperature at $85 \mathrm{~K}$. Macroscopic quantities such as density, temperature, and velocity fields were calculated using control volumes to confirm that the system reached an equilibrium state. The dimensions of the control volumes were $L_{x} \times L_{y} \times \Delta z \mathrm{~nm}$, where $\Delta z=0.1 \mathrm{~nm}$.

Figs. 2(b) and 3(b) show the density, temperature, and velocity fields of $\mathrm{Ar}$ and $\mathrm{Ne}$ molecules in the calculation systems with $N_{\mathrm{Ne}}=4,000$ and 12,000 , respectively. The velocity fields were calculated by adding average molecular velocities in the $x$-, $y$-, and $z$ directions. Thus, the velocity fields should be zero over the entire system when the molecules are in equilibrium. The figures confirm that the temperature is uniform at $T=85 \mathrm{~K}$, and the velocity is uniform at $0 \mathrm{~m} / \mathrm{s}$ for both $\mathrm{Ar}$ and Ne molecules. This indicates that the system had reached the equilibrium state. We established equilibrium systems in all four cases of $N_{\mathrm{Ne}}$.

Figs. 2(c) and 3(c) show the density fields in the range $2 \leq z \leq 8$ in the cases of $N_{\mathrm{Ne}}=4,000$ and 12,000 , respectively. The density field of Ne molecules shows a peak near the gas-liquid interface, indicating that $\mathrm{Ne}$ molecules form an adsorption film on the interface. The density of the adsorption film increases as $N_{\mathrm{Ne}}$ increases, and the same tendency for the formation of the adsorption films has been reported in previous studies [14,21]. In addition, the density of Ne molecules in the liquid phase increases with an increase in $N_{\mathrm{Ne}}$ as well as in the gas phase. This indicates that the dissolved amount of $\mathrm{Ne}$ molecules increases owing to the increase in their partial pressure in the gas phase.

\subsection{Classification of molecular motions}

$J_{\text {evap }}^{V}$ and $J_{\text {out }}^{V *}$ must be obtained for the calculation of $\alpha_{\mathrm{e}}^{V}$, as given by Eq. (1). To separately obtain these molecular mass fluxes, molecules must be classified into evaporation, condensation, and reflection molecules, as shown in Fig. 1. Thus, to classify molecules moving in the vicinity of the gas-liquid interface, we used a method involving setting two boundaries in the current MD simulations. This classification method has been proposed by Meland et al. [31] and $\mathrm{Gu}$ et al. [32], and it has been improved by 
(a)

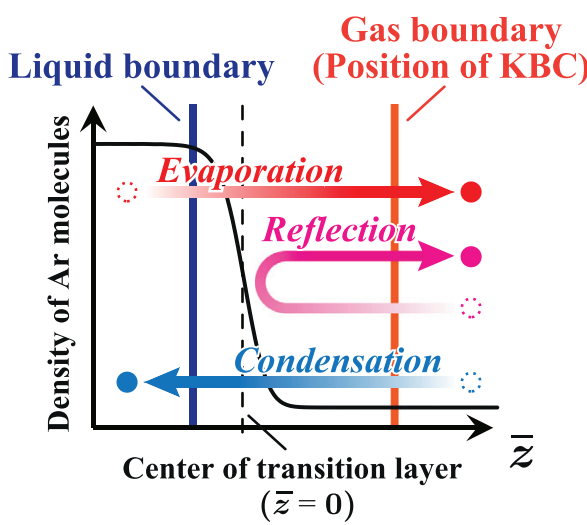

(b)

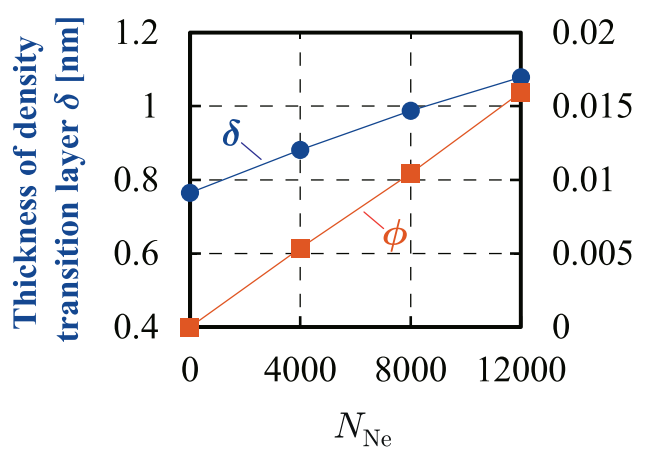

Fig. 4. (a) Schematic of classification method of molecules that involves setting two boundaries. (b) Thickness of the density transition layer $\delta$ and molar fraction $\phi$ in each case of $N_{\mathrm{Ne}}$

Kobayashi et al. [17] for calculating $\alpha_{\mathrm{e}}^{V}$ and $\alpha_{\mathrm{c}}^{V}$ in the binary mixture system.

The concept of the method involving setting the two boundaries-the liquid boundary and the gas boundary-is shown in Fig. 4(a). Using this method, molecules are classified into evaporation, condensation, and reflection molecules based on the following definitions. First, a molecule that passes through two boundaries from the liquid phase to the gas phase is defined as an evaporation molecule. Second, a molecule that passes through two boundaries from the gas phase to the liquid phase is defined as a condensation molecule. Finally, a molecule that passes through the gas boundary from the gas phase and returns to the gas phase without passing through the liquid boundary is defined as a reflection molecule.

The positions to set the two boundaries are determined based on the following normalized $z$ coordinate:

$\bar{z}=\frac{z-Z_{\mathrm{m}}}{\delta}$,

where $\delta$ denotes the 10-90 thickness of the density transition layer, and $Z_{\mathrm{m}}$ denotes the position of the center of the transition layer. $\delta$ and $Z_{\mathrm{m}}$ can be obtained from the following hyperbolic tangent function $[6,33]$ :

$\rho(z)=\frac{\rho_{v}+\rho_{l}}{2}+\frac{\rho_{v}-\rho_{l}}{2} \tanh \left(\frac{z-Z_{\mathrm{m}}}{0.455 \delta}\right)$,

where $\rho_{v}$ and $\rho_{l}$ denote the densities of $\mathrm{Ar}$ molecules in the gas and liquid phases, respectively. It is known that $\delta$ depends on dimensions of a calculation system, liquid temperature, and a presence of NC gas molecules $[6,14,21]$. The relation between $N_{\mathrm{Ne}}$ and $\delta$ herein is shown in Fig. 4(b). The figure confirms that $\delta$ increases as $N_{\mathrm{Ne}}$ increases, which is consistent with the tendency reported in $[14,21]$.
Since the position of the gas boundary is where the molecular mass fluxes and $\alpha_{\mathrm{e}}^{V}$ are calculated, the positions of the gas boundary and the KBCs including $\alpha_{\mathrm{e}}^{V}$ should coincide. Thus, we set the gas boundary at $\bar{z}=3.0$, which has already been determined as the position for setting KBCs in the field of MGD [11].

In contrast to the gas boundary, there is no unified definition of where to set the liquid boundary. Moreover, although the position of the liquid boundary was proposed in [17] by referring to the mass flux of evaporation vapor molecules under the virtual vacuum condition $[6,8]$, the validity of that position had not been verified. Thus, in our previous study [34], the appropriate position of the liquid boundary was discussed based on the balance between the time that vapor molecules colliding with the gas-liquid interface stay around the interface and their reached position to the liquid-phase side. Based on the results obtained in [34], we set the liquid boundary at $\bar{z}=-1.0$, which is the position where molecules that stay around the interface for a short time and immediately return to the gas phase are properly classified as reflection molecules.

As $\alpha_{\mathrm{e}}^{V}$ has been shown as a function of the molar fraction of NC gas molecules in the liquid phase in the binary mixture system [14,21], we organized $\alpha_{\mathrm{e}}^{V}$ and molecular mass fluxes using the molar fraction $\phi$, given by

$\phi=\frac{N^{G}}{N^{V}+N^{G}}$,

where $N^{V}$ and $N^{G}$ denote the numbers of vapor (Ar) and NC gas $(\mathrm{Ne})$ molecules in the bulk liquid phase $(\bar{z}<-1.0)$, respectively. The relation between $N_{\mathrm{Ne}}$ and $\phi$ obtained in the current MD study is shown in Fig. 4(b). The values of $\phi$ corresponding to the cases where $N_{\mathrm{Ne}}=0,4,000,8,000$, and 12,000 are $\phi=0.0,0.0054$, 0.0104 , and 0.0159 , respectively.

\subsection{Establishment of non-equilibrium system}

The calculation system becomes a non-equilibrium state where the net evaporation occurs when the evaporation amount is greater than the condensation amount. To establish such a nonequilibrium system, in this study, we intentionally removed $\mathrm{Ar}$ molecules from the calculation system in the gas phase. This prevents vapor molecules from condensing; thus, the condensation amount becomes smaller than the evaporation amount, and net evaporation occurs.

To quantitatively determine the non-equilibrium of the calculation system, the following vapor density ratio $\chi$ is defined:

$\chi=\frac{\rho_{v}}{\rho_{v}^{*}\left(T_{\mathrm{L}}\right)}$,

where $\rho_{v}$ denotes the vapor density in the gas phase, and $\rho_{v}^{*}\left(T_{\mathrm{L}}\right)$ denotes the saturated vapor density at the liquid temperature $T_{\mathrm{L}}$. In this study, we defined the region outside the gas boundary $(\bar{z}>3.0)$ as the gas phase. In the equilibrium state, where the evaporation amount and the condensation amount are the same, $\rho_{v}$ is equal to $\rho_{v}^{*}$ and the vapor density ratio is $\chi=1$. In the case of $\chi<1$, the system is in a non-equilibrium state, where the net evaporation occurs as the condensation amount is lower than that in the equilibrium state owing to the lower vapor density $\rho_{v}$ than the saturated vapor density $\rho_{v}^{*}\left(T_{\mathrm{L}}\right)$.

The definition of $\chi$ given by Eq. (8) indicates that the system at $\chi=0$ is the limit case of the non-equilibrium state in which net evaporation occurs. Thus, $\alpha_{\mathrm{e}}^{V}$ in each case of $N_{\mathrm{Ne}}$ was calculated in the range $0 \leq \chi \leq 1$. Simulations in the systems at $\chi=0$ and 1 were performed to analyze and discuss the results of the current MD study, and simulations at $\chi=0.5$ were also performed as a supplement to the results.

To produce a non-equilibrium system at $\chi=0$, all $\mathrm{Ar}$ (vapor) molecules in the gas phase $(\bar{z}>3.0)$ in the initial composition were 

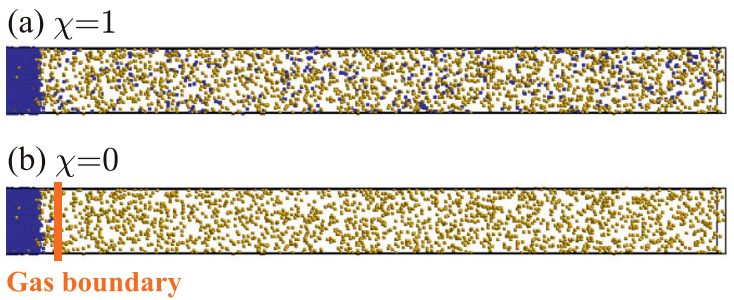

Fig. 5. Calculation systems with $N_{\mathrm{Ne}}=4,000$ at (a) $\chi=1$ and (b) $\chi=0$. There are no $\mathrm{Ar}$ molecules (blue spheres) in the gas phase when the system is in a nonequilibrium state at $\chi=0$. (For interpretation of the references to colour in this figure legend, the reader is referred to the web version of this article.)

removed from the calculation system to reduce $\rho_{v}$ in the initial condition to zero. After the simulations started, Ar molecules that evaporated from the liquid phase to the gas phase were also removed to maintain $\rho_{v}$ of the system at zero. When producing the system at $\chi=0.5, \rho_{v}$ in the initial condition was reduced to half of $\rho_{v}^{*}$ by randomly removing half of the $\mathrm{Ar}$ molecules in the gas phase, and one in two evaporation Ar molecules was removed from the calculation system in the order in which they passed through the gas boundary throughout the simulation to maintain $\chi=0.5$. Since the present study was based on long simulation times in the system with the periodic boundary conditions imposed, no significant variations of the state of Ar molecules in the gas phase could occur in the non-equilibrium system at $\chi=0.5$.

Fig. 5 shows the calculation systems with $N_{\mathrm{Ne}}=4,000$ in the equilibrium state at $\chi=1$ and in the limit case of the nonequilibrium state at $\chi=0$. The figure confirms that there are no Ar molecules in the gas phase outside the gas boundary in the calculation system at $\chi=0$.

The intentional removal of evaporation $\mathrm{Ar}$ molecules passing through the gas boundary might affect the motions of $\mathrm{Ne}$ molecules in the gas phase, especially in the vicinity of the gas boundary. However, in the current MD simulations, the velocity scaling method was applied to $\mathrm{Ne}$ molecules in the gas phase when the system was in a non-equilibrium state, and the velocity distribution for colliding Ne molecules with the gas-liquid interface was controlled to remain constant (details are provided in Sections 2.4 and 3.1). Therefore, the removal of evaporation molecules from the calculation system had no effect on the $\mathrm{Ne}$ molecules.

The evaporation $\mathrm{Ar}$ molecules were removed from the calculation system one after another in non-equilibrium systems. Owing to the decrease in the number of Ar molecules composing the liquid phase, the gas-liquid interface receded from its original position during net evaporation. According to the recession of the interface, the positions of the liquid and gas boundaries had to be renewed to calculate the molecular mass fluxes. Thus, we adopted the following position of the center of the density transition layer considering the movement of the gas-liquid interface [6]:

$Z_{\mathrm{m}}^{t+\Delta t}=Z_{\mathrm{m}}^{t}-v_{\mathrm{e}} \Delta t, v_{\mathrm{e}}=\frac{J_{\mathrm{e}}}{\rho_{\mathrm{l}}}$,

where $Z_{\mathrm{m}}^{t}$ denotes the position of the center of the density transition layer at time $t$, and $J_{\mathrm{e}}$ denotes the mass flux of the evaporation molecules calculated at each time step $\Delta t$. When the system is in the non-equilibrium state at $\chi<1$, the positions of the liquid and gas boundaries were renewed at every time step based on the calculated $Z_{\mathrm{m}}^{t+\Delta t}$. As the receding length in each simulation was sufficiently short compared to the $z$-directional length of the gas phase in the calculation system, the change in the partial pressure of $\mathrm{Ne}$ molecules caused by the receding of the interface was negligibly small.
Furthermore, the net evaporation leads the decrease in the liquid temperature. When calculating the molecular mass fluxes and $\alpha_{\mathrm{e}}^{V}$ via time averaging under a certain constant condition, causing steady and constant evaporation throughout the simulations is necessary. Thus, in the case of the non-equilibrium state $(\chi<1)$, the velocity scaling method was applied to molecules in the bulk liquid region within $\bar{z}<-2.0$ to maintain the liquid temperature at $T_{\mathrm{L}}=85 \mathrm{~K}$.

When the width of the temperature control region in the liquid phase became less than $2 \delta$ owing to the recession of $Z_{\mathrm{m}}^{t}$, we stopped the simulation of the non-equilibrium system. This is because we considered that the thickness of the liquid film became insufficient for accurate calculations. The stoppage of the simulations caused the small number of sample molecules for the calculations of molecular mass fluxes and the evaporation coefficient $\alpha_{\mathrm{e}}^{V}$. This makes it impossible to accurately calculate them in a single simulation. To perform sampling with a sufficient number of molecules in the non-equilibrium system, we ran simulations using more than 10 different initial arrangements of molecules in all cases of $N_{\mathrm{Ne}}$ and $\chi$.

\subsection{State of NC gas molecules in the non-equilibrium system}

The steady and net evaporation caused by the reduction in the vapor density ratio $\chi$ is the main topic of this study. As described in the previous subsection, the liquid temperature was maintained at $T_{\mathrm{L}}=85 \mathrm{~K}$, and the number of vapor molecules in the gas phase was controlled to maintain $\chi$ at a constant value. However, NC gas molecules were not desired to vary their influence on the net evaporation of vapor molecules throughout the simulations.

To make the influence of $\mathrm{NC}$ gas $(\mathrm{Ne})$ molecules on the evaporation constant, their states in the vicinity of the gas-liquid interface must not change. Factors of Ne molecules that can affect the evaporation of vapor molecules include density, temperature, and velocity distribution around the interface. These can be kept constant when the velocity distribution of colliding Ne molecules from the gas phase and their densities remain the same as those in the equilibrium state. By keeping Ne molecules in the gas phase in the equilibrium state at $85 \mathrm{~K}$, the density and velocity distribution of colliding Ne molecules can remain constant even during net evaporation.

From the above, we lengthened the $z$-directional system length $L_{z}$ to five times larger than that in our previous study [14], and we applied temperature control for Ne molecules in the gas phase to bring them into equilibrium. Lengthening $L_{z}$ increases the number of collisions between Ne molecules in the gas phase, thereby making it easier for the gas phase to reach the equilibrium state. The velocity scaling method was applied to Ne molecules in $\bar{z}>5.0$ to maintain their temperature at $T=85 \mathrm{~K}$ when the system was in a non-equilibrium state.

To quantitatively evaluate whether the state of $\mathrm{Ne}$ molecules in the gas phase could reach the equilibrium state, the Knudsen number in the gas phase was calculated. The Knudsen number, $\mathrm{Kn}=\lambda / \mathrm{L}$, is a dimensionless number defined as the ratio of the mean free path of molecules $\lambda$ to the characteristic length of a system $L$. In general, molecules are considered to be in equilibrium when $\mathrm{Kn} \ll 1$. $L_{z}$ of the calculation system corresponds to the characteristic length $L$ in the denominator of $\mathrm{Kn}$. The mean free path $\lambda$ can be estimated by

$\lambda=\frac{1}{\sqrt{2} \pi \sigma^{2} n}$,

where $\sigma$ denotes the molecular diameter and $n$ denotes the number density of molecules in the gas phase.

In the case of $N_{\mathrm{Ne}}=4,000$, which has the smallest number of $N_{\mathrm{Ne}}$ in the vapor-gas binary system in this study, the Kn of $\mathrm{Ne}$ 
(a) $\phi=0.0054$

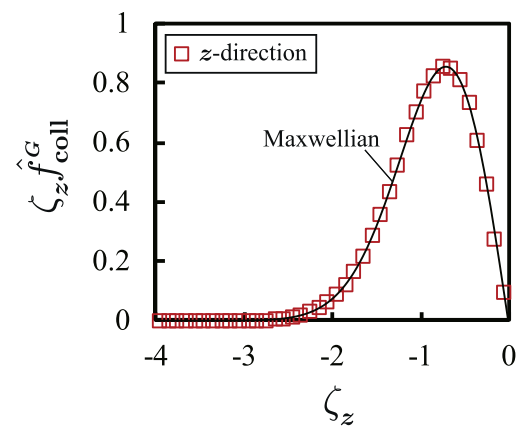

(c) $\phi=0.0054$

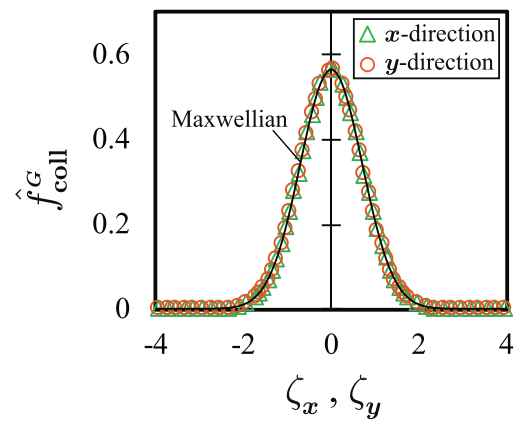

(b) $\phi=0.0159$

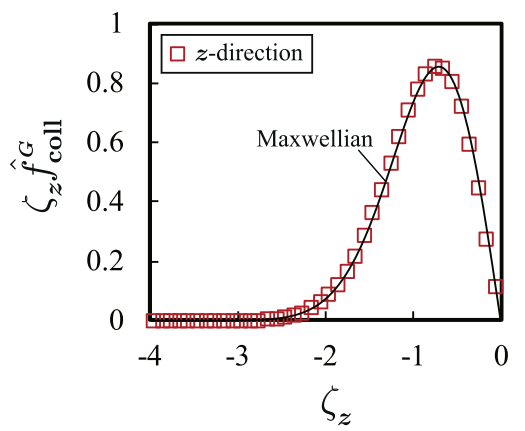

(d) $\phi=0.0159$

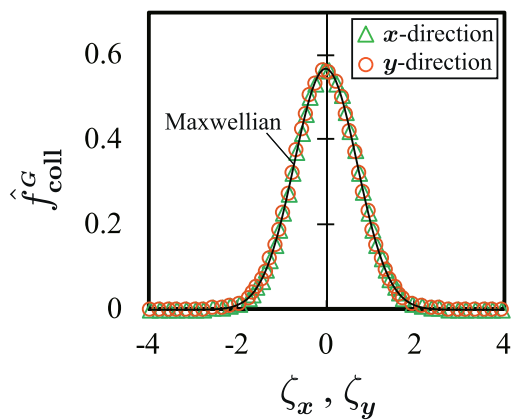

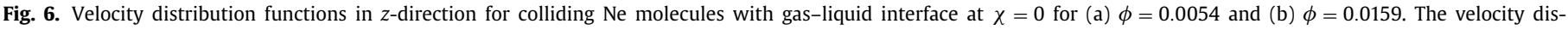

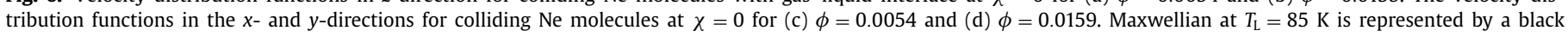
solid line.

molecules was calculated as $\mathrm{Kn}=0.0511$. The applied parameters of $\sigma, n$, and $L$ for calculating this Kn were $\sigma=\sigma_{\mathrm{Ne}}=0.275 \mathrm{~nm}$, $n=0.364 \mathrm{~nm}^{-3}$, and $L=16.0 \mathrm{~nm}$, respectively. The characteristic length $L=16.0 \mathrm{~nm}$ represents the approximate length of the gas phase in $L_{z}$ of the calculation system excluding the thickness of the liquid film. As we consider $\mathrm{Kn}=0.0511$ as $\mathrm{Kn} \ll 1$, Ne molecules in the gas phase were assumed to maintain the equilibrium state during the net evaporation of vapor molecules. When $\mathrm{Ne}$ molecules in the gas phase are in an equilibrium state, they collide with the gas-liquid interface while following a constant velocity distribution with a constant density according to their temperature at $T=85 \mathrm{~K}$. This would maintain the states of $\mathrm{Ne}$ molecules in the vicinity of the gas-liquid interface constant. The validity of this assumption is verified at the beginning of the next section.

\section{Results and discussion}

\subsection{State of Ne molecules during net evaporation}

Fig. 6 shows the velocity distribution functions of Ne molecules colliding with the gas-liquid interface in the non-equilibrium state at $\chi=0$ in the cases of $\phi=0.0054$ and 0.0159 (the cases of $N_{\mathrm{Ne}}=4,000$ and 12,000). The velocity distribution function in the $z$-direction is shown as a function $\zeta_{z} \hat{f}_{\text {coll }}^{G}$ in Fig. $6(\mathrm{a})$ and (b). $\hat{f}_{\text {coll }}^{G}$ denotes the normalized velocity distribution function of colliding Ne molecules, and $\zeta_{z}$ denotes the normalized molecular velocity in the $z$-direction given by $\zeta_{z}=\xi_{z} / \sqrt{2 R^{G} T_{\mathrm{L}}}$, where $R^{G}$ denotes the gas constant of NC gas molecules and $\sqrt{2 R^{G} T_{\mathrm{L}}}$ denotes the most probable speed of them in the Maxwellian at the liquid temperature $T_{\mathrm{L}}$. The $z$-directional Maxwellian, which is represented by the solid line in Fig. 6(a) and (b), is given by

$\zeta_{z} \hat{f}_{z}^{*}=2 \zeta_{z} \exp \left(-\zeta_{z}^{2}\right)$
In addition, the velocity distribution functions in the $x$ - and $y$ directions are shown as a normalized form $\hat{f}_{\text {coll }}^{G}$ in Fig. $6(\mathrm{c})$ and (d). $\zeta_{x}$ and $\zeta_{y}$ in the abscissa denote the molecular velocities in the $x$ - and $y$-directions normalized to $\sqrt{2 R^{G} T_{\mathrm{L}}}$, respectively. The $x$ or $y$-directional Maxwellian, which is represented by the solid line in Fig. 6(c) and (d), is given by

$\hat{f}_{j}^{*}=\frac{1}{\sqrt{\pi}} \exp \left(-\zeta_{j}^{2}\right), \quad j=x, y$.

As shown in Fig. 6, the velocity distributions in all directions agree well with the Maxwellian in both cases of $\phi$. This indicates that Ne molecules maintained their velocity distributions at those in the equilibrium state (i.e., Maxwellian at $85 \mathrm{~K}$ ) even when the system was in the non-equilibrium state at $\chi=0$, where the net evaporation of vapor molecules occurs. Not only in the cases shown in Fig. 6, the agreements of the velocity distribution functions with the Maxwellian were confirmed under all conditions of $\phi$ and $\chi$ herein.

Furthermore, Fig. 7 shows the density fields of $\mathrm{Ar}$ and $\mathrm{Ne}$ molecules in the systems at $\chi=1$ (the equilibrium state) and $\chi=$ 0 (the limit case of the non-equilibrium state). The density fields in the cases of $\phi=0.0054,0.0104$, and 0.0159 are shown in Fig. 7(a)(c), and the density field in the low-density range $\rho<80.0 \mathrm{~kg} / \mathrm{m}^{3}$ for $\phi=0.0054$ is shown in Fig. 7(d). The solid lines represent the density fields of the $\mathrm{Ar}$ and Ne molecules at $\chi=1$, whereas the open squares represent those at $\chi=0$.

In all cases of $\phi$ in Fig. 7(a)-(c), the density fields show almost the same profiles regardless of the value of $\chi$. As there are no condensation vapor molecules from the gas phase in the nonequilibrium system at $\chi=0$, the density field of $\mathrm{Ar}$ molecules around the gas boundary $(1<\bar{z}<3)$ becomes lower than that at $\chi=1$, as shown in Fig. 7(d). However, Fig. 7 fundamentally confirms that the states around the gas-liquid interface in $\bar{z}<1$ and $\mathrm{Ne}$ molecules between the liquid and gas boundaries maintain certain constant states during the net evaporation of vapor molecules. 
(a) $\phi=0.0054$

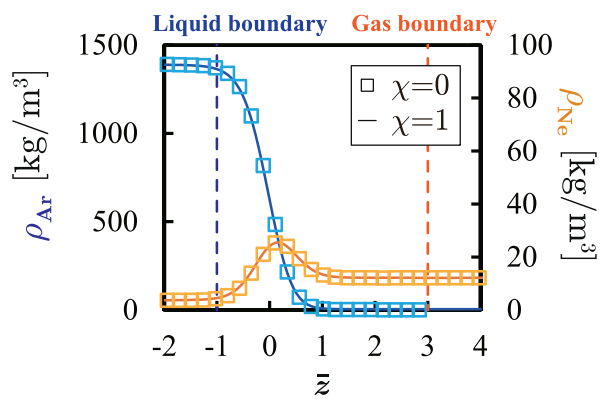

(c) $\phi=0.0159$

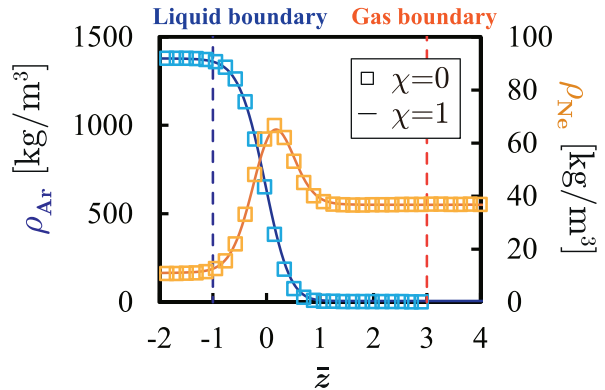

(b) $\phi=0.0104$

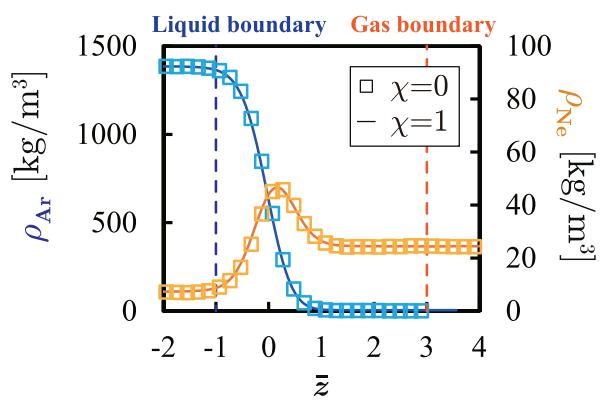

(d) $\phi=0.0054, \rho<80.0 \mathrm{~kg} / \mathrm{m}^{3}$

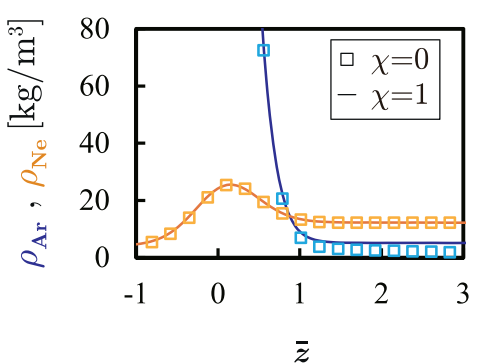

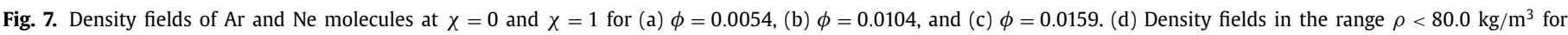
$\phi=0.0054$. Open squares represent density fields at $\chi=0$, and solid lines represent those at $\chi=1$.

As shown in Figs. 6 and 7, the velocity distribution function of colliding Ne molecules and the profiles of the density fields around the gas-liquid interface in the non-equilibrium system do not change from those in the equilibrium system. This indicates that there was no difference between the equilibrium and the non-equilibrium states except for the number of condensation molecules from the gas phase. Therefore, we conclude that the influence of Ne molecules on the motions of Ar molecules around the interface did not change throughout the simulations, and the steady net evaporation in the non-equilibrium system was primarily caused by the reduction in the vapor density ratio $\chi$ in the current MD simulations.

Furthermore, the result shown in Fig. 6 also indicates that the removal of $\mathrm{Ar}$ molecules did not disturb the state of Ne molecules in the gas phase in the non-equilibrium system, which provides some assurance of the validity of the method to establish the nonequilibrium system.

\subsection{Evaporation coefficient and mass fluxes of vapor molecules}

Fig. 8 shows the evaporation coefficient $\alpha_{\mathrm{e}}^{V}$ of vapor molecules in each case of the vapor density ratio $\chi$ and the molar fraction $\phi$. The abscissa represents $\chi$ and the ordinate represents $\alpha_{\mathrm{e}}^{V}$. Dashed line represents the average value of $\alpha_{\mathrm{e}}^{V}$ in three cases of $\chi$. The figure confirms that $\alpha_{\mathrm{e}}^{V}$ takes an almost constant value regardless of the value of $\chi$ in each case of $\phi$, whereas $\alpha_{\mathrm{e}}^{V}$ decreases as $\phi$ increases in each case of $\chi$. This indicates that $\alpha_{\mathrm{e}}^{V}$ is independent of whether the system is in an equilibrium or a nonequilibrium state, whereas it greatly depends on the molar fraction $\phi$. Since $\chi=0$ is the limit case of the non-equilibrium state where net evaporation occurs, the figure illustrates that $\alpha_{\mathrm{e}}^{V}$ takes almost the same value in any non-equilibrium state within the range $0 \leq \chi<1$ for an arbitrary number of Ne molecules in the system. Before elucidating on the causes of the tendencies shown in Fig. 8, we next show the molecular mass fluxes because $\alpha_{\mathrm{e}}^{V}$ is calculated from $J_{\text {evap }}^{V}$ and $J_{\text {out }}^{V *}$.

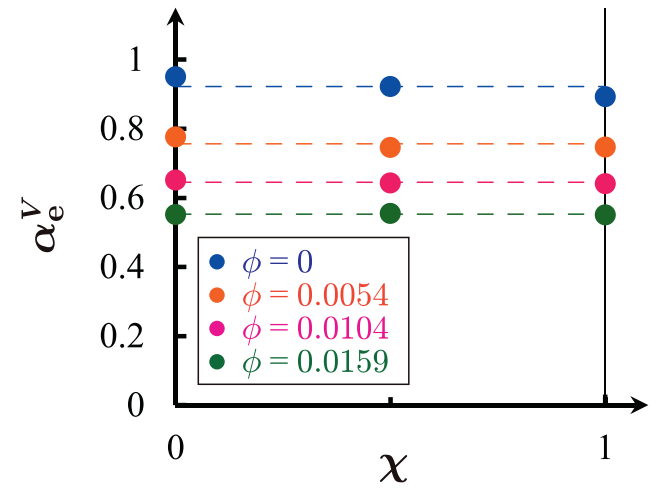

Fig. 8. Evaporation coefficient $\alpha_{\mathrm{e}}^{V}$ in $0 \leq \chi \leq 1$ in each case of $\phi$.

The mass fluxes of Ar molecules at $\chi=0$ and 1 are shown in Fig. 9(a) and (b), respectively. The abscissa represents $\phi$ and the ordinate represents the value of mass fluxes. As the reflection of $\mathrm{Ar}$ molecules does not occur in the non-equilibrium system at $\chi=0$, the mass flux of reflection $\operatorname{Ar}$ molecules $J_{\text {ref }}^{V}$ is zero in all cases of $\phi$ in Fig. 9(a). In contrast, $J_{\text {ref }}^{V}$ in the equilibrium system at $\chi=$ 1 increases as $\phi$ increases, indicating that colliding $\mathrm{Ar}$ molecules from the gas phase to the gas-liquid interface get reflected more easily owing to the increase in the number of Ne molecules in the system.

The mass flux of the evaporation Ar molecules $J_{\text {evap }}^{V}$ decreases as $\phi$ increases in both cases of $\chi$. Furthermore, the figures confirm that $J_{\text {evap }}^{V}$ has almost the same value regardless of the value of $\chi$. From the definition of $\alpha_{\mathrm{e}}^{V}$, given by Eq. (1), $J_{\text {out }}^{V *}$ in the equilibrium state are used to calculate $\alpha_{\mathrm{e}}^{V}$ in both the equilibrium and nonequilibrium states. Hence, $J_{\text {evap }}^{V}$, which does not change depending on $\chi$, makes $\alpha_{\mathrm{e}}^{V}$ the same value regardless of the value of $\chi$ (i.e., the system state) in each case of $\phi$. The factors that cause $J_{\text {evap }}^{V}$ to have the same value regardless of the value of $\chi$ are discussed in 
(a) $\chi=0$

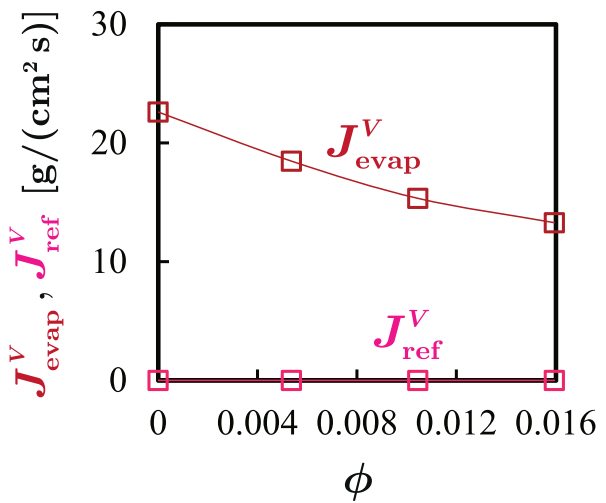

(b) $\chi=1$
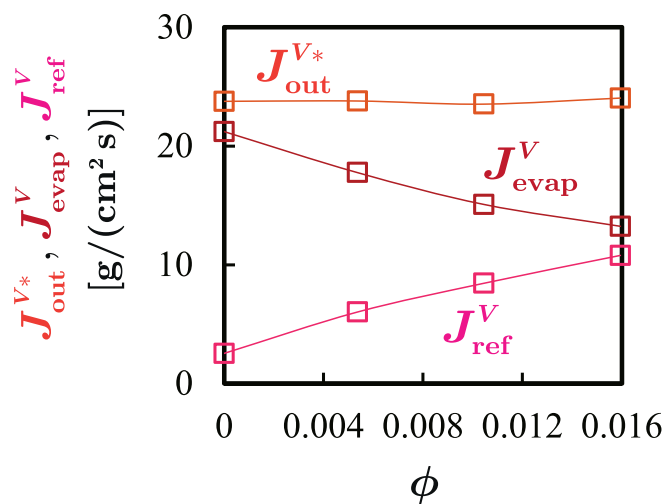

Fig. 9. Mass fluxes of $\operatorname{Ar}$ molecules at (a) $\chi=0$ and (b) $\chi=1$.

Section 3.3, and the detailed influences of $\phi$ on $J_{\text {evap }}^{V}$ are discussed in Section 3.4.

Although it is not the main issue of this study, the condensation coefficient $\alpha_{c}^{V}$ is also calculated for $\chi=0.5$ and 1 . The condensation coefficient $\alpha_{\mathrm{c}}^{V}$ was reported to have almost the same value as the evaporation coefficient $\alpha_{\mathrm{e}}^{V}$ in the equilibrium and binary mixture system [14,21], and the same tendency is observed in the case of $\chi=1$ in this study. In addition, the value of $\alpha_{c}^{V}$ in the nonequilibrium system at $\chi=0.5$ does not change from that in the equilibrium system at $\chi=1$. This result suggests that $\alpha_{\mathrm{c}}^{V}$ is also independent of the system state, as in the case of the evaporation coefficient $\alpha_{\mathrm{e}}^{V}$.

The condensability of vapor molecules has been reported to depend on their collision velocity toward the gas-liquid interface [34-36], which means that the value of $\alpha_{c}^{V}$ is affected by the velocity distribution of them. In the current MD simulations, the velocity distribution of the colliding vapor molecules remained constant regardless of the system state as well as that of the NC gas molecules, and thus, $\alpha_{\mathrm{c}}^{V}$ is independent of whether the system is in an equilibrium or a non-equilibrium state.

\subsection{Influence of system state on evaporation of vapor molecules}

The difference between the equilibrium and the nonequilibrium states in the current MD study is the presence or absence of condensation vapor molecules from the gas phase. As already shown in Fig. $7(\mathrm{~d})$, the vapor density around the gas boundary in the non-equilibrium state at $\chi=0$ becomes lower than that in the equilibrium state at $\chi=1$ owing to the absence of (a)
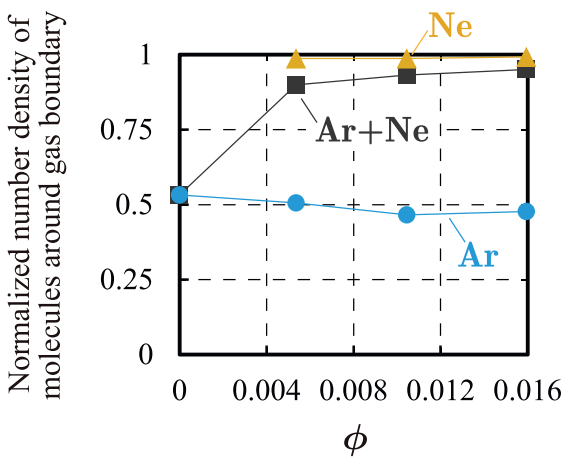

(b)

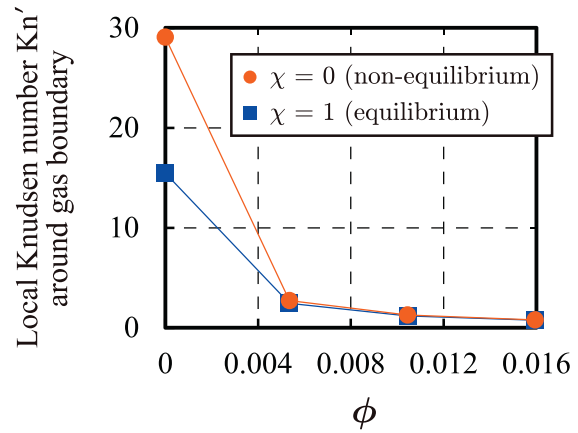

Fig. 10. (a) Normalized number densities of $\mathrm{Ar}$, $\mathrm{Ne}$, and sum of $\mathrm{Ar}$ and $\mathrm{Ne}$ molecules in $1<\bar{z}<3$ for each $\phi$. Number density of molecules at $\chi=0$ is normalized to that at $\chi=1$. (b) Local Knudsen numbers in $1<\bar{z}<3$ at $\chi=1$ and 0 for each $\phi$.

condensation vapor molecules. As the molecular number density corresponds to the molecular collision rate [1], the change in the molecular density around the gas boundary can affect the ease of evaporation of vapor molecules from the liquid phase to the gas phase. Specifically, a higher number density of molecules causes more molecular collisions, and vapor molecules that attempt to evaporate are more likely to be prevented from evaporating via collisions.

Fig. 10(a) shows the normalized number densities of $\mathrm{Ar}$ molecules, Ne molecules, and the sum of $\mathrm{Ar}$ and Ne molecules around the gas boundary in the range $1<\bar{z}<3$ in each case of $\phi$. The ordinate represents the number density in the non-equilibrium state at $\chi=0$ normalized to that in the equilibrium state at $\chi=1$. The figure indicates that the normalized number density of $\mathrm{Ar}$ molecules in all cases of $\phi$ is approximately 0.5 , indicating that the number density of vapor molecules at $\chi=0$ is almost half of that at $\chi=1$ around the gas boundary. In contrast, the normalized number density of Ne molecules is almost unity because the density profile of Ne molecules is almost independent of the system state, as shown in Fig. 7. Owing to the unchanged number density of Ne molecules, the normalized number density of the sum of $\mathrm{Ar}$ and Ne molecules in the cases of $\phi \neq 0$ approaches unity.

To correlate the number density of molecules with the molecular collision rate around the gas boundary, we calculate a local Knudsen number, $\mathrm{Kn}^{\prime}$, in the range $1<\bar{z}<3$. As described in Section 2.4, the Knudsen number represents the ratio of the mean free path of molecules to the characteristic length. Thus, we can estimate the molecular collision rate within an arbitrary region by calculating $\mathrm{Kn}^{\prime}$.

Fig. 10(b) shows the $\mathrm{Kn}^{\prime}$ in the range $1<\bar{z}<3$ in the equilibrium state at $\chi=1$ and the non-equilibrium state at $\chi=0$. The thickness of the density transition layer $\delta$ and the number den- 
sity of the sum of Ar and Ne molecules in the range $1<\bar{z}<3$ in each case of $\phi$ are applied to the characteristic length $L$ and the number density $n$ for the calculation of $\mathrm{Kn}^{\prime}$, respectively. The figure indicates that $\mathrm{Kn}^{\prime}$ differs considerably between the equilibrium state at $\chi=1$ and the non-equilibrium state at $\chi=0$ only in the case of $\phi=0$ as the number density of molecules around the gas boundary greatly differs in this case, as shown in Fig. 10(a).

However, $\mathrm{Kn}^{\prime}$ in the case of $\phi=0$ in the equilibrium state at $\chi=1$ is approximately 15 , and it is known that a high Knudsen number, generally 10 or more, represents the free-molecular flow wherein molecular collisions seldom occur [3]. This means that when the single-component system is in the equilibrium state, the evaporation of vapor molecules is hardly hindered by molecular collisions in the first place, although the molecular number density and collision rate around the gas boundary are higher than those in the non-equilibrium state. From the above, $J_{\text {evap }}^{V}$ and the corresponding $\alpha_{\mathrm{e}}^{V}$ are independent of whether the system is in equilibrium or non-equilibrium (i.e., the value of $\chi$ ) in the singlecomponent system of $\phi=0$.

In the case of $\phi \neq 0$, which represents the vapor-gas binary mixture system, $\mathrm{Kn}^{\prime}$ in the range $1<\bar{z}<3$ takes almost the same value between the equilibrium state at $\chi=1$ and the nonequilibrium state at $\chi=0$. This is because the number density of Ne molecules around the gas boundary is independent of the system state, as shown in Fig. 10(a). The fact that $\mathrm{Kn}^{\prime}$ does not change indicates that the molecular collision rate does not change. We conclude that in the case of $\phi \neq 0$, the possibility that evaporation of vapor molecules is hindered by molecular collisions does not change with the system state (i.e., the value of $\chi$ ); therefore, $J_{\text {evap }}^{V}$ and the corresponding $\alpha_{\mathrm{e}}^{V}$ in the binary mixture system take almost the same value regardless of whether the system is in equilibrium or non-equilibrium.

In this subsection, we demonstrate that $\alpha_{\mathrm{e}}^{V}$ is independent of the system state. Subsequently, we demonstrate the influence of the molar fraction $\phi$ of NC gas molecules on $J_{\text {evap }}^{V}$ and $\alpha_{\mathrm{e}}^{V}$.

\subsection{Influence of NC gas molecules on evaporation of vapor molecules}

Based on the discussion in the previous subsection, the decrease in $J_{\text {evap }}^{V}$ with the increase in $\phi$ shown in Fig. 9 can be expected to be caused by the change in the number density of NC gas molecules around the gas-liquid interface, which is related to the molecular collision rate. Furthermore, as Ne molecules form an adsorption film on the interface, the influence of Ne molecules on $J_{\text {evap }}^{V}$ could change depending on the position around the interface. Thus, we investigate where their influence appears prominently as well as the factor that causes the decrease in $J_{\text {evap. Note }}^{V}$ that, since it has been shown in the previous subsection that the system state-the value of $\chi$-has no effect on the evaporation of Ar molecules, the investigation in the following is conducted using the case $\chi=0$ as an example.

To that end, we defined a backscattering molecule, as shown in Fig. 11(a). An Ar molecule that passed through the liquid boundary from the liquid phase and returned to it again without passing through the gas boundary was defined as the backscattering molecule. In addition, the maximum $\bar{z}$ position where a backscattering molecule reached was defined as $z_{\text {reach }}$. Regions where Ne molecules dominantly reduce $J_{\text {evap }}^{V}$ could be estimated from the tendency of $z_{\text {reach }}$ of backscattering molecules in each case of $\phi$.

Fig. 11(b) shows the frequency distribution of $z_{\text {reach }}$ with the interval $\Delta z_{\text {reach }}=0.5 . z_{\text {reach }}$ of the backscattering molecules in each case of $\phi$ were obtained within a sampling time of 10 ns in the non-equilibrium system at $\chi=0$. The ordinate represents the number of backscattering molecules in each interval normalized to the total number of them obtained during the sampling time in the case of $\phi=0$. The inset of the figure shows the frequency distribu- (a)

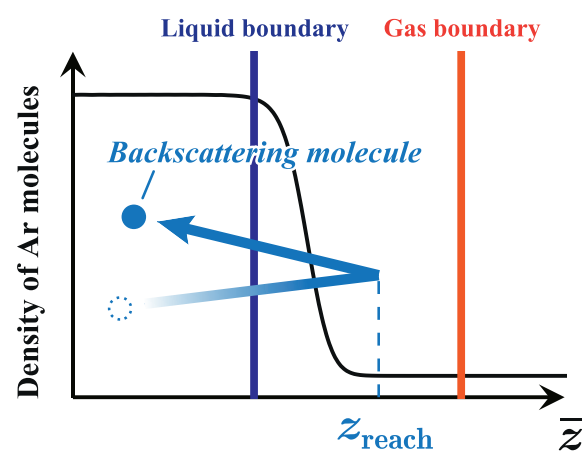

(b)

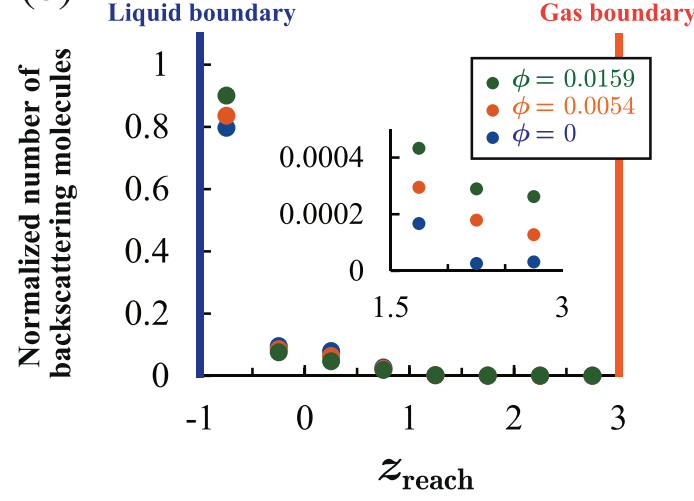

Fig. 11. (a) Schematic of backscattering molecule and $z_{\text {reach }}$ (b) Frequency distribution of $z_{\text {reach }}$ between the liquid and gas boundaries with the interval $\Delta z_{\text {reach }}=0.5$.

tion in the range $1.5<z_{\text {reach }}<3.0$. The result of $\phi=0$ in the figure represents the number of backscattering molecules which was caused by the interaction of only Ar molecules because there were no Ne molecules in the system. Thus, the result in this case does not include the influence of Ne molecules, and the difference between the result of $\phi=0$ and other cases of $\phi$ is caused by the presence of Ne molecules.

As shown in Fig. 11(b), most molecules are backscattered in the range $\bar{z}<1.0$ in all cases of $\phi$. This indicates that a large number of backscattering molecules returned to the liquid phase from around the gas-liquid interface. In contrast, although the number of backscattering molecules is miniscule in the region around the gas boundary, the inset of the figure illustrates that the number of backscattering molecules has more than doubled between $\phi=0$ and 0.0159 . This result suggests that while the number of backscattering molecules around the gas boundary is small, the influence of the increase in $\phi$ on backscattering is larger around the gas boundary than around the gas-liquid interface.

To elucidate the influence of $\phi$ on backscattering occurring around the liquid boundary side and the gas boundary side, we investigated the rate of increase in the number of backscattering molecules with the increase in $\phi$ on those two sides. The range $-1 \leq \bar{z}<1$ was defined as the liquid-phase subregion, and the range $1 \leq \bar{z}<3$ was defined as the gas-phase subregion.

Fig. 12(a) shows the normalized number of backscattering molecules in the liquid-phase subregion and gas-phase subregion in each case of $\phi$. The number of backscattering molecules is normalized to that at $\phi=0$ in each subregion. As shown in the figure, the number of backscattering molecules increases as $\phi$ increases on both the liquid-phase and gas-phase subregions. The increase in the number of backscattering molecules indicates that the evaporation of vapor molecules becomes more hindered. Therefore, the decrease in $J_{\text {evap }}^{V}$ with an increase in $\phi$ shown in Fig. 9 was 
(a)

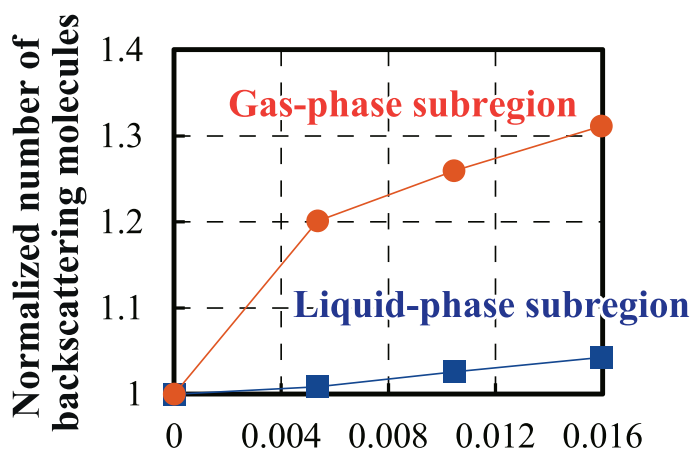

$\phi$

(b)

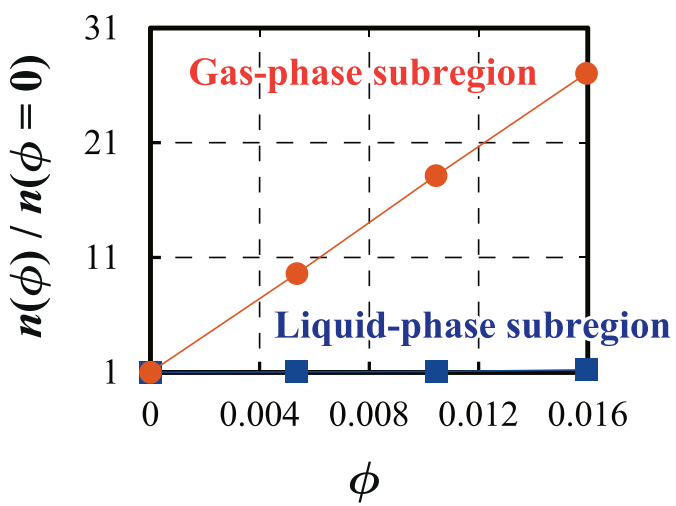

Fig. 12. (a) Normalized number of backscattering molecules and (b) normalized number density of molecules in the liquid-phase and gas-phase subregions in each case of $\phi$.

found to be caused by vapor molecules becoming more likely to be backscattered throughout the entire region between the liquid and gas boundaries. However, the figure confirms that the normalized number of backscattering molecules in the gas-phase subregion greatly increases compared to that in the liquid-phase subregion. This indicates that $\phi$ has a greater influence on the backscattering of vapor molecules in the gas-phase subregion.

We next elucidate the reason why the influence of $\phi$ on the backscattering is greater in the gas-phase subregion side than in the liquid-phase subregion. In a previous study [14], the number density of molecules in the range $\bar{z}<1.0$ was investigated to evaluate the relation between the molecular collisions and evaporation mass fluxes in a binary mixture system. However, the investigation of the number density in the range $\bar{z}>1.0$, where an increase in the number of backscattering molecules is large has not been made. Thus, we calculated and compared the number density of molecules on the liquid-phase and the gas-phase subregions.

The number densities $n(\phi)$ in the liquid-phase and gas-phase subregions were calculated from the total number of both $\mathrm{Ar}$ and Ne molecules in the ranges $-1 \leq \bar{z}<1$ and $1 \leq \bar{z}<3$, respectively. Because the adsorption film of Ne molecules was formed around $\bar{z} \approx 0$, as shown in Fig. 7, its influence on the backscattering can be estimated from $n(\phi)$ in the liquid-phase subregion.

Fig. 12(b) shows the normalized number density of molecules in the liquid-phase and the gas-phase subregions in each case of $\phi$. The number density $n(\phi)$ is normalized to that at $\phi=0$ on each side. In the gas-phases subregion, the normalized number density at $\phi=0.0159$ is approximately 27 times larger than that at $\phi=0$. In contrast, in the liquid-phase subregion, the normalized number density at $\phi=0.0159$ is approximately 1.2 times larger than that at $\phi=0$. This indicates that the increase in the number density with the increase in $\phi$ is more remarkable in the gas-phase subregion than in the liquid-phase subregion. It was also confirmed that the adsorption film of Ne molecules, which has a locally high density around the gas-liquid interface, does not significantly increase $n(\phi)$ in the liquid-phase subregion.

A higher number density of molecules causes more molecular collisions, as mentioned in the previous subsection. Therefore, we conclude that a large increase in the backscattering molecules in the gas-phase subregion shown in Fig. 10(a) is caused by a large increase in the molecular collision rate owing to the higher number density of the molecules. This is the reason that the influence of the increase in $\phi$ on the backscattering is greater in the gasphase subregion than in the liquid-phase subregion.

\subsection{Velocity distribution function of evaporation molecules}

Fig. 13 shows the velocity distribution functions for evaporation Ar molecules $\hat{f}_{\text {evap }}^{V}$ in the $z$-direction in each case of $\phi$. The velocity distributions in the cases of $\chi=0$ and 1 are shown as a function $\zeta_{z} \hat{f}_{\text {evap }}^{V}$, and the $z$-directional Maxwellian represented by the solid line is given by $\zeta_{z} \hat{f}_{z}^{*}$. The figure illustrates that regardless of the value of $\chi$, the velocity distributions show an almost constant distribution in each case of $\phi$. Thus, $\hat{f}_{\text {evap }}^{V}$ is independent of whether the system is in an equilibrium or a non-equilibrium state.

The figure also indicates that the mean velocity becomes higher as $\phi$ increases. This indicates that evaporation vapor molecules tend to have a higher velocity as the number of NC gas molecules in the system increases. The increase in the mean velocities with the increase in $\phi$ has also been reported in a previous MD study of binary mixtures and equilibrium systems [14]; however, the factors that cause this tendency have not been elucidated. Hence, we discuss the cause of the increase in the mean velocity of $\hat{f}_{\text {evap }}^{V}$.

Fig. 14 shows the attractive force acting on an Ar molecule on the $\bar{z}$ coordinate in the system in each case of $\phi$. The force was calculated based on the attractive term in the Lennard-Jones potential function given by Eq. (3). The negative values on the ordinate represent the force toward the liquid-phase side-the force acting in the negative direction of the $\bar{z}$ coordinate. Although the attractive forces in the figure were calculated in the equilibrium system, the same profiles of them would be obtained in the non-equilibrium system because the state of the gas-liquid interface hardly changes according to the system state herein (Fig. 7).

The figure confirms that a large force in the negative $\bar{z}$ direction acts on a molecule in the vicinity of the gas-liquid interface in all cases of $\phi$. This is owing to the intermolecular force from molecules composing the liquid phase. In addition, a force in the positive $\bar{z}$ direction acts in the cases of $\phi=0.0054$ and 0.0159 around $\bar{z}<-1.0$ owing to the intermolecular interaction with $\mathrm{Ne}$ molecules composing the adsorption film.

It has been reported that the attractive force toward the liquidphase side acting on molecules in the vicinity of the interface contributes to the formation of the adsorption film of NC gas molecules [14,21]. In addition, the velocity of evaporation vapor molecules is decelerated by this attractive force toward the liquidphase side before the molecules reach the gas boundary. As shown in Fig. 14, the attractive force acting in the vicinity of the interface weakens as $\phi$ increases. This is because the thickness of the transition layer increases with an increase in $\phi$, as shown in Fig. 4(b). As $\phi$ increases and the attractive force toward the liquid-phase side weakens, the deceleration of the rate of evaporation molecules is suppressed. This leads to the conclusion that the weakening of the attractive force is one of the causes of the increase in the mean velocity of $\hat{f}_{\text {evap }}^{V}$ with an increase in $\phi$. 

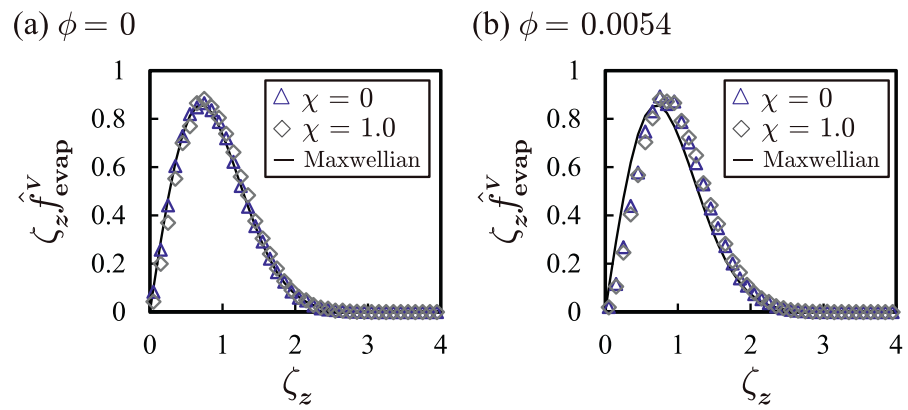

(c) $\phi=0.0159$

Fig. 13. Velocity distribution functions for evaporation $\operatorname{Ar}$ molecules for (a) $\phi=0$, (b) $\phi=0.0054$, and (c) $\phi=0.0159$.

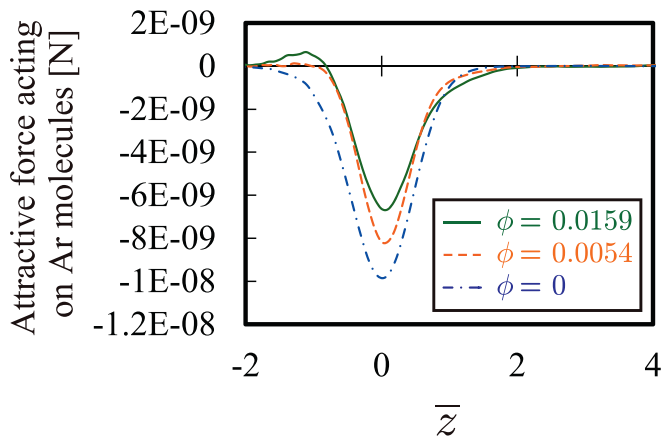

Fig. 14. Attractive force acting on $\mathrm{Ar}$ molecule on $\bar{z}$ coordinate. A negative value indicates aa force acting in the negative direction of $\bar{z}$.

\section{Conclusion}

The evaporation coefficient $\alpha_{\mathrm{e}}^{V}$ during steady net evaporation was calculated using MD simulations in a vapor-gas binary mixture system. We proposed a calculation system that can perform net evaporation calculations up to the extreme case of nonequilibrium, and investigated in detail the values of $\alpha_{\mathrm{e}}^{V}$ and the evaporation mechanisms over a wide range of parameters. We have observed that $\alpha_{\mathrm{e}}^{V}$ in any non-equilibrium state where the net evaporation occurs takes almost the same value as that in the equilibrium state. As the number density of NC gas molecules in the vicinity of the gas-vapor interface is irrelevant to the system state in the current MD simulations, the collision rate of molecules around the interface hardly changes. This indicates that the probability that the evaporation of vapor molecules is hindered by molecular collisions does not change between the equilibrium state and the non-equilibrium state in the binary mixture system; as a result, $\alpha_{\mathrm{e}}^{V}$ takes almost the same values in the two states.

It was also observed that $\alpha_{\mathrm{e}}^{V}$ decreases as the molar fraction of the NC gas molecules increases. This decrease in $\alpha_{\mathrm{e}}^{V}$ has been clarified to be caused by the increase in the probability of collisions with NC gas molecules that prevent vapor molecules from evaporating. In particular, the influence of the increase in the molar fraction of NC gas molecules on the evaporation of vapor molecules is larger around the gas phase in the vicinity of the gas-liquid interface than around the liquid phase.

Furthermore, the mean velocity of the velocity distribution function of evaporation vapor molecules increases as the molar fraction increases, whereas the profile of the velocity distribution function is independent of whether the system is in equilibrium or non-equilibrium. The increase in the molar fraction increases the thickness of the gas-liquid interface, thereby weakening the attractive force acting between evaporation vapor molecules and molecules around the interface. Thus, we consider that the weakening of the attractive force acting on evaporation vapor molecules is one of the factors that causes an increase in the mean velocity of the evaporation molecules.

\section{Declaration of Competing Interest}

The authors declare the following financial interests/personal relationships which may be considered as potential competing interests:

Hirofumi Tabe reports financial support was provided by Japan Society for the Promotion of Science. Kazumichi Kobayashi reports financial support was provided by Japan Science and Technology Agency. Kazumichi Kobayashi reports financial support was provided by Japan Society for the Promotion of Science. Kazumichi Kobayashi reports financial support was provided by f3 Engineering Education and Research Center, Faculty of Engineering, Hokkaido University.

\section{CRediT authorship contribution statement}

Hirofumi Tabe: Conceptualization, Data curation, Formal analysis, Funding acquisition, Investigation, Methodology, Software, Validation, Visualization, Writing - original draft, Writing - review \& editing. Kazumichi Kobayashi: Conceptualization, Funding acquisition, Investigation, Methodology, Project administration, Resources, Software, Supervision, Validation, Writing - review \& editing. Hiroyuki Fujii: Supervision, Writing - review \& editing. Masao Watanabe: Supervision, Writing - review \& editing.

\section{Acknowledgments}

This study was partly supported by Research Fellowships of Japan Society for the Promotion of Science (JSPS) for Young Scientists (21J10669) to H.T., JST, PRESTO Grant Number JPMJPR2103, Japan to K.K., JSPS KAKENHI Grant Number 20K04277 to K.K., and f3 Engineering Education and Research Center, Faculty of Engineering, Hokkaido University to K.K.

\section{References}

[1] G.A. Bird, J. Brady, Molecular Gas Dynamics and the Direct Simulation of Gas Flows, vol. 5, Clarendon Press Oxford, 1994.

[2] C. Cercignani, Rarefied Gas dynamics: From Basic Concepts to Actual Calculations, vol. 21, Cambridge University Press, 2000.

[3] Y. Sone, Molecular Gas Dynamics: Theory, Techniques, and Applications, Springer Science \& Business Media, 2007.

[4] M. Matsumoto, Molecular dynamics of fluid phase change, Fluid Phase Equilib. $144(1-2)(1998)$ 307-314.

[5] T. Tsuruta, H. Tanaka, T. Masuoka, Condensation/evaporation coefficient and velocity distributions at liquid-vapor interface, Int. J. Heat Mass Transf. 42 (22) (1999) 4107-4116.

[6] T. Ishiyama, T. Yano, S. Fujikawa, Molecular dynamics study of kinetic boundary condition at an interface between argon vapor and its condensed phase, Phys. Fluids 16 (8) (2004) 2899-2906.

[7] M. Bond, H. Struchtrup, Mean evaporation and condensation coefficients based on energy dependent condensation probability, Phys. Rev. E 70 (6) (2004) 061605. 
[8] T. Ishiyama, T. Yano, S. Fujikawa, Molecular dynamics study of kinetic boundary condition at an interface between a polyatomic vapor and its condensed phase, Phys. Fluids 16 (12) (2004) 4713-4726.

[9] T. Ishiyama, T. Yano, S. Fujikawa, Kinetic boundary condition at a vapor-liquid interface, Phys. Rev. Lett. 95 (8) (2005) 084504.

[10] A. Kryukov, V.Y. Levashov, About evaporation-condensation coefficients on the vapor-liquid interface of high thermal conductivity matters, Int. J. Heat Mass Transf. 54 (13-14) (2011) 3042-3048.

[11] S. Fujikawa, T. Yano, M. Watanabe, Vapor-Liquid Interfaces, Bubbles and Droplets: Fundamentals and Applications, Springer Science \& Business Media, 2011.

[12] M. Kon, K. Kobayashi, M. Watanabe, Method of determining kinetic boundary conditions in net evaporation/condensation, Phys. Fluids 26 (7) (2014) 072003.

[13] M. Kon, K. Kobayashi, M. Watanabe, Liquid temperature dependence of kinetic boundary condition at vapor-liquid interface, Int. J. Heat Mass Transf. 99 (2016) 317-326.

[14] K. Kobayashi, K. Sasaki, M. Kon, H. Fujii, M. Watanabe, Kinetic boundary conditions for vapor-gas binary mixture, Microfluid Nanofluidics 21 (3) (2017) 53.

[15] S. Anisimov, D. Dunikov, V. Zhakhovskii, S. Malyshenko, Properties of a liquid-gas interface at high-rate evaporation, J. Chem. Phys. 110 (17) (1999) 8722-8729.

[16] A. Frezzotti, L. Gibelli, S. Lorenzani, Mean field kinetic theory description of evaporation of a fluid into vacuum, Phys. Fluids 17 (1) (2005) 012102.

[17] K. Kobayashi, K. Hori, M. Kon, K. Sasaki, M. Watanabe, Molecular dynamics study on evaporation and reflection of monatomic molecules to construct kinetic boundary condition in vapor-liquid equilibria, Heat Mass Transf. 52 (9) (2016) 1851-1859.

[18] M. Kon, K. Kobayashi, M. Watanabe, Kinetic boundary condition in vapor-liquid two-phase system during unsteady net evaporation/condensation, Eur. J. Mech.-B/Fluids 64 (2017) 81-92.

[19] A. Frezzotti, L. Gibelli, D.A. Lockerby, J.E. Sprittles, Mean-field kinetic theory approach to evaporation of a binary liquid into vacuum, Phys. Rev. Fluids 3 (5) (2018) 054001

[20] S. Busuioc, L. Gibelli, D.A. Lockerby, J.E. Sprittles, Velocity distribution function of spontaneously evaporating atoms, Phys. Rev. Fluids 5 (10) (2020) 103401.

[21] K. Ohashi, K. Kobayashi, H. Fujii, M. Watanabe, Evaporation coefficient and condensation coefficient of vapor under high gas pressure conditions, Sci. Rep. 10 (1) (2020) 1-10.

[22] Z. Liang, P. Keblinski, Molecular simulation of steady-state evaporation and condensation in the presence of a non-condensable gas, J. Chem. Phys. 148 (6) (2018) 064708.
[23] J. Gonzalez, J. Ortega, Z. Liang, Prediction of thermal conductance at liquid-gas interfaces using molecular dynamics simulations, Int. J. Heat Mass Transf. 126 (2018) 1183-1192.

[24] E. Bird, J.G. Plascencia, P. Keblinski, Z. Liang, Molecular simulation of steady-state evaporation and condensation of water in air, Int. J. Heat Mass Transf. 184 (2022) 122285.

[25] A. Frezzotti, Non-equilibrium structure of the vapor-liquid interface of a binary fluid, in: AIP Conference Proceedings, vol. 1333, American Institute of Physics, 2011, pp. 161-166.

[26] K. Kobayashi, T. Nagayama, M. Watanabe, H. Fujii, M. Kon, Molecular gas dynamics analysis on condensation coefficient of vapour during gas-vapour bubble collapse, J. Fluid Mech. 856 (2018) 1045-1063.

[27] V.Y. Levashov, A.P. Kryukov, I.N. Shishkova, Influence of the noncondensable component on the characteristics of temperature change and the intensity of water droplet evaporation, Int. J. Heat Mass Transf. 127 (2018) 115-122.

[28] K. Yamamoto, K. Kobayashi, M. Watanabe, H. Fujii, M. Kon, H. Takahira, Influence of a small amount of noncondensable gas on shock wave generation inside a collapsing vapor bubble, Phys. Rev. Fluids 4 (6) (2019) 063603.

[29] V.G. Baidakov, S.P. Protsenko, Molecular-dynamics investigation of phase equilibrium and surface tension in argon- neon system, J. Phys. Chem. C 112 (44) (2008) 17231-17234.

[30] M.P. Allen, D.J. Tildesley, Computer Simulation of Liquids, Oxford University Press, 2017.

[31] R. Meland, A. Frezzotti, T. Ytrehus, B. Hafskjold, Nonequilibrium molecular-dynamics simulation of net evaporation and net condensation, and evaluation of the gas-kinetic boundary condition at the interphase, Phys. Fluids 16 (2) (2004) 223-243.

[32] K. Gu, C.B. Watkins, J. Koplik, Molecular dynamics simulation of the equilibrium liquid-vapor interphase with solidification, Fluid Phase Equilib. 297 (1) (2010) 77-89.

[33] J. Lekner, J. Henderson, Theoretical determination of the thickness of a liquidvapour interface, Physica A 94 (3-4) (1978) 545-558.

[34] H. Tabe, K. Kobayashi, H. Fujii, M. Watanabe, Molecular dynamics study on characteristics of reflection and condensation molecules at vapor-liquid equilibrium state, PLoS ONE 16 (3) (2021) e0248660.

[35] T. Tsuruta, G. Nagayama, Molecular dynamics studies on the condensation coefficient of water, J. Phys. Chem. B 108 (5) (2004) 1736-1743.

[36] A. Tokunaga, T. Tsuruta, Nonequilibrium molecular dynamics study on energy accommodation coefficient on condensing liquid surface-molecular boundary conditions for heat and mass transfer, Phys. Fluids 32 (11) (2020) 112011. 